\title{
Neuroscience tools: brain insights
}

\author{
Nathan Blow
}

\begin{abstract}
Researchers at two Boston-based neuroscience centers are working to develop new imaging tools and technology with the hope of discovering the secrets behind how the brain functions.
\end{abstract}

During the week Harvard University neuroscientist Randy Buckner travels several times between his two Boston-area offices, one at the Martinos Center for Biomedical Imaging in Charlestown and the other across the river in Cambridge on the main Harvard University campus. At the Martinos Center-where researchers are using a host of different imaging modalities, from magnetic resonance imaging (MRI) to positron emission tomography (PET), to explore the human brain- he serves as the center's associate director. At Harvard University, he is affiliated with both the psychology department and the newly created Center for Brain Science, where advanced imaging approaches are being used on everything from humans to fruit flies in an effort to better understand the basics of brain anatomy and function. "I would love for all of this to be in the same building, but I do not think that exists anywhere," comments Buckner.

\section{Aligning protons}

When it comes to noninvasive, in vivo imaging of the brain, the MRI instruments that Buckner nd his colleagues use have few peers. At the heart of MRI technology is a large, usually cylindrical magnet, ranging in strength from 0.35 to 15 tesla $(\mathrm{T})$, which serves to align the protons found in water throughout the body. A smaller, radiofrequency electromagnetic field is then used to push some protons out of alignment. When the protons are displaced from their equilibrium position they precess, emitting a small radiofrequency signal characteristic of their environment that can be used to generate an image.

Most advances in MRI technology occur in an area of instrument design where the equation is simple: increase the field strength of the magnet and this increases the spatial resolution of the imaging scan. Cutting-edge

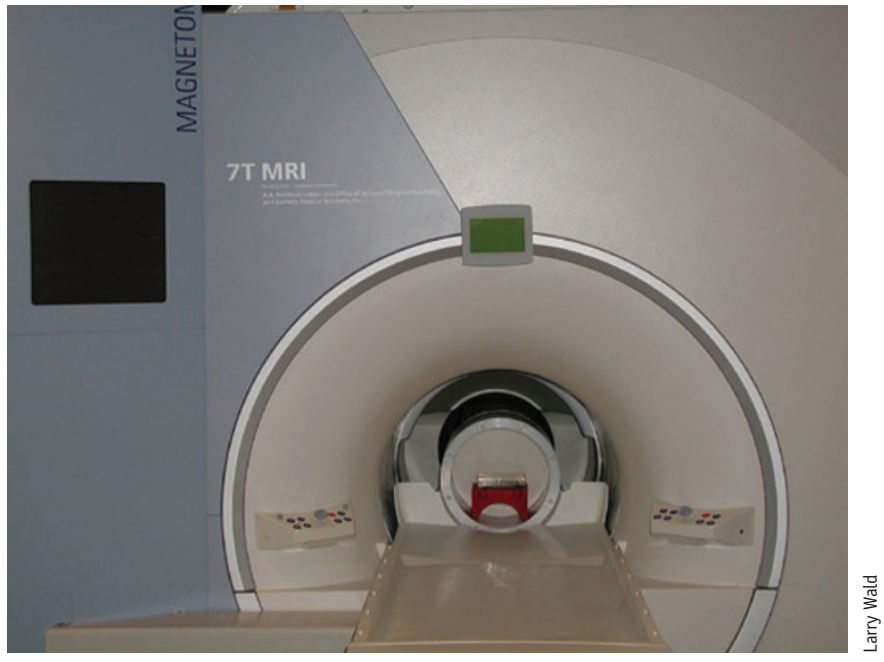

A 7T MRI system for human imaging applications.

research MRI instruments used for smallanimal imaging can have field strengths of 9.4-15 T, but most clinical MRI instruments used for human brain imaging range in strength from 1.5-3 T, although topof-the-line research grade $7 \mathrm{~T}$ systems are also available from companies like Siemens Healthcare in Malvern, Pennsylvania, USA, GE Healthcare in Piscataway, New Jersey, USA and Philips in Andover, Massachusetts, USA.

The Martinos Center has nine MRI systems used for both clinical and research applications. And on this September afternoon a technician was working to ramp up the center's latest addition, a new state-of-theart $15 \mathrm{~T}$ magnet designed by Varian of Palo Alto, California, USA and Magnex Scientific in Walnut Creek, California, USA for smallanimal imaging. Larry Wald, director of the center's NMR core, says using a magnet of this strength will provide researchers with more detailed images of mouse brain anatomy and could yield new insights into neural disease progression in animal models.
Having a high-strength magnet to align protons is only the first step in MRI; those protons still need to be excited and their oscillating fields need to be detected for any real picture to emerge. Ten years ago, MRI instruments relied on radiofrequency transmission systems with single coils for excitation and detection of protons. Today most instruments come equipped with multiple channel detection coils that allow the parallel acquisition of proton data during a scan, resulting in improved sensitivity and considerably shorter scan times.

When it comes to the development of new detection coils, Wald says Martinos Center researchers have made an effort to push both the technology and the commercial developers forward. "We have tried to egg the developers on in certain directions," he acknowledges with a smile.

Wald's group has already designed a variety of different detection coils for specific imaging applications, including a 32-channel brain coil developed for the 


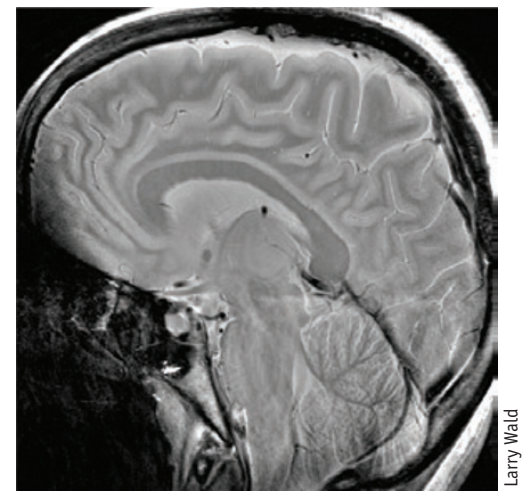

Human anatomical image acquired with 7 T MRI.

Siemens $7 \mathrm{~T}$ human MRI system and a 96-channel brain-specific coil for signal detection in Siemens clinical $3 \mathrm{~T}$ MAGNETOM. They also work on custom coil designs for more specific applications, such as one researcher's request to be able to better image a patient's carotid arteries for which Wald's group designed detection coils that would fit around the neck.

"We can provide the designs and initial validation, which can save a company years in development time," explains Wald. His efforts helped Siemens develop technologies such as Total imaging matrix — which allows coil arrangements to be changed depending on imaging needs in a configuration of up to 32 radiofrequency channels — and other coil technology for MRI instrumentation.

\section{Faster images, more function}

Neuroscientists are seeing the benefit of the better spatial resolution provided by stronger magnets and the faster scanning capabilities in multichannel detection systems. "We have protocols now where we can get a reasonable amount of anatomical and functional information in about 15 minutes," says Buckner. And this is not only valuable for clinical brain imaging applications, but it is also advancing research into how the brain actually functions.

Functional MRI (fMRI) is a technique that looks at brain function by measuring changes in the amounts of oxygenated and deoxygenated blood as a marker for neural activity. As it is noninvasive, $\mathrm{fMRI}$ studies on everything from the cognitive basis for risk-taking behavior to how neurological diseases such as Alzheimer's progress in individual brains have been reported in recent years as more and more researchers gain access to advanced MRI technology.

"Ten years ago when we were doing neuroimaging we were looking at groups of people because the methods did not have enough power to see and make maps of individual brains," notes Buckner. But today he says they can get a good battery of measurements on anatomy of the brain, morphometry and functional anatomy for an individual during a single scan.

For researchers in Buckner's group, these developments are making it possible to finally explore links between an individual's brain phenotype and their underlying genetic composition. Buckner's goal is to use neuroimaging to look at individual brains, generate maps of the structure, connections and function, and then use genomic approaches, such as genomewide association studies or whole-genome resequencing, to correlate a given brain phenotype to a specific genotype. And at only 15 minutes for a scan, Buckner sees a tremendous possibility for addressing many different questions in neuroscience using MRI. "If you can add on something as short as 15 minutes to get information to pool across studies, you have a real opportunity to take advantage of the

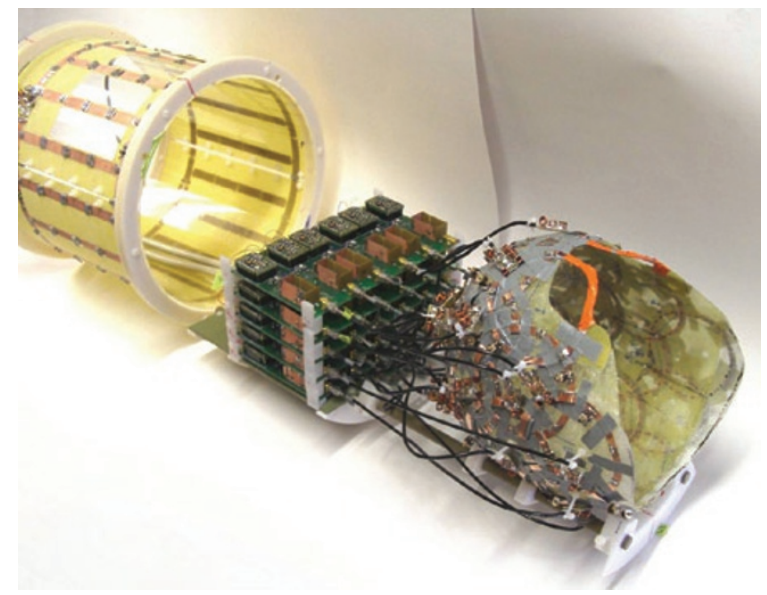




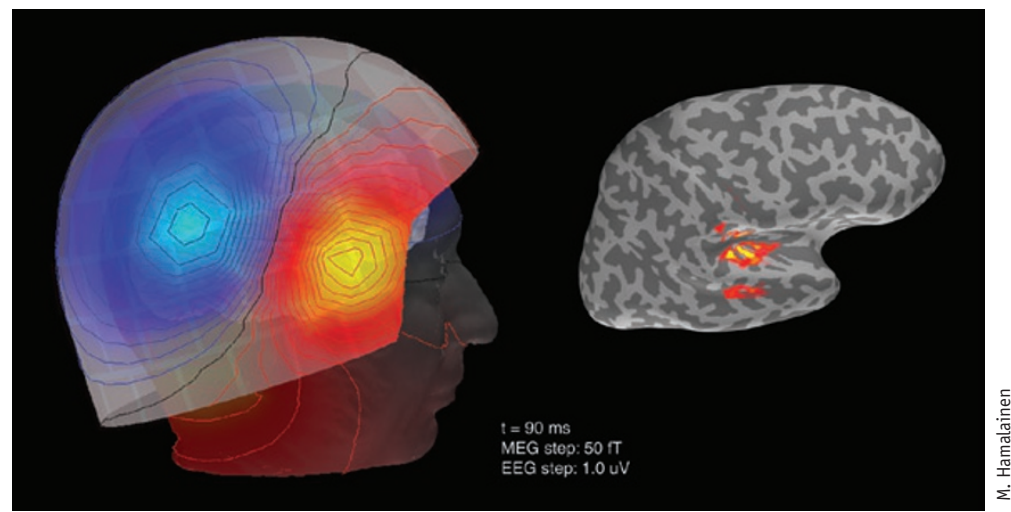

MEG can be used to follow the timing of brain activation with excellent temporal resolution. Shown are a topographical map of acquired MEG signals 90 milliseconds after stimulation (left) and an estimation of corresponding neural current (right).

large number of [patients] going through Harvard and Martinos to develop a largescale resource where you have information on thousands of brains and genetics."

\section{MEG speed}

Matti Hamalainen, director of the magnetoencephalography (MEG) core at the
Martinos Center, analyzes magnetic signals induced by synchronized local neuronal currents from the cortex of the human brain that are a billionth the strength of the earth's magnetic field. "It is like hearing a needle dropping on a pillow inside a loud disco," explains Hamalainen as he stands in a large metal room containing the MEG instrument. The walls are about a foot thick and composed of layers of different metals that act to eliminate all outside magnetic fields, allowing the instrument to sense the weak signals generated from brain activity.

The Neuromag MEG instrument from Elekta-Neuromag (Helsinki, Finland) at the center of the room looks like a reclining metal chair, albeit with an enormous helmet attached at the top. This helmet actually contains 306 superconducting liquid helium-cooled sensors that circle the head, each recording a time series of magnetic signals from a subject's brain. MEG instruments are rare: the Martinos Center's single MEG instrument is one of only three in the New England area and one of less than 40 throughout the United States.

Although the sensors in the MEG helmet provide very good temporal resolution of localized electrical activity in the brain cortex, the spatial resolution of the technique is lower than that of fMRI. For this reason, Hamalainen's group has been developing software that can integrate anatomical MRI information with his group's MEG data to 
more accurately map electrical changes to specific regions of the cortex.

Still, when it comes to functional studies, it turns out the high temporal resolution of the MEG electrical scanning approach complements the better spatial resolution of fMRI's slower blood flow analysis quite well. In fact, by combining data from both approaches, an even clearer picture of neural activation can often emerge.

\section{Big picture}

Sitting in his office at the Martinos Center, Bruce Fischl, director of the computational core, has a very simple explanation for the complex job he has at the center: "I build models of the brain."

Fischl's models attempt to integrate the anatomical information provided by MRI with functional data to both define specific areas and boundaries within the brain and correlate that information with a region's function. The functional data can come from fMRI, from MEG and from optical techniques such as the diffusion optical imaging approach being advanced by David Boas and the Martinos Center's optical imaging core. But even with four sources of data, the major challenge Fischl faces with his models is resolution.

Anatomically, MRI scans using the best magnets and coils can get down to 300micrometer resolution, a far cry from conventional optical microscopy's resolution of 200-450 nanometers, where individual neurons can be seen. "We are not going to get to 5 [micrometers] in the next 5 to 10 years," suspects Fischl. At the moment it is not even clear how whole-brain neuroimaging could get to that magical micrometer level of resolution. Most researchers say it is not clear yet whether advances in the existing technology will be adequate or whether new technology development will be necessary to achieve such resolution. But Fischl and others do know they will have to figure out how to avoid blurring effects in neuroimaging data to move forward. At millimeter resolution - typically the resolution used for clinical brain imaging - blurring caused by simple natural events like patient motion or normal cardiac and respiratory cycles is not a problem. But at 100micrometer resolution, those biological events can play havoc with imaging and the subsequent data analysis. Fischl notes that the two-photon microscopy optical imaging approach Boas' group has developed to visualize brain vasculature is very effective at measuring physiological activity in the brain, and he is hopeful that this technique can be used to locate and remove some of the blurring in MRI data.

There are other ways to avoid blurring at higher resolution. "Using ex vivo tissue samples we are not far from that level of resolution," notes Fischl. Wald's group of coil developers has constructed a specific set of coils for imaging a single human brain ex vivo in an MRI instrument. "We were able to almost shrink-wrap the coils to the brain, giving a boost in sensitivity," explains Wald. And with the added ability to image for up to 12 hours without any movement or other blurring

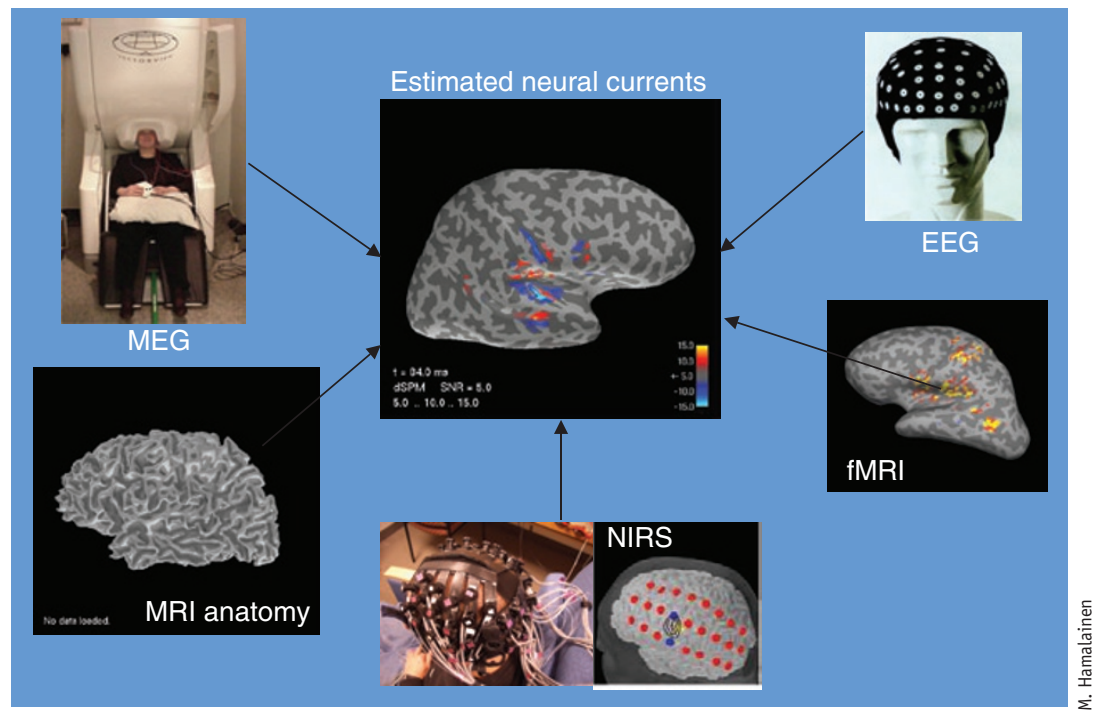

The Martinos Center relies on the integration of data from multiple imaging modalities, including MRI, MEG, electroencephalography (EEG) and near-infrared spectroscopy (NIRS). 

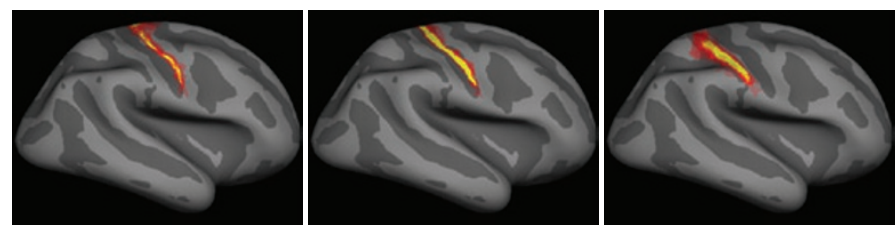

$0 \%$
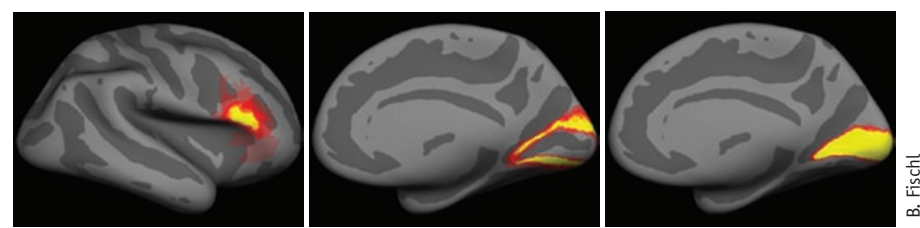

Models of the brain based on measurements of cortical geometry show ability to predict specific brain regions.

factors, the resolution of ex vivo imaging is much better than that in vivo, allowing Fischl to map these results back to lower-resolution in vivo images.

Still, the disparity between looking at the cellular level and what can be obtained using human neuroimaging approaches is enormous. "A great challenge for neuroscience is bridging the chasm between [magnetic resonance] and optical resolution," says Jeff Lichtman from Harvard University, who is also a faculty member at the Center for Brain Sciences.

At the cellular level, Lichtman and others at the Center for Brain Science are working to reconstruct wiring diagrams of the brain at micrometer-level single-neuron resolution. For Lichtman, reconstructing the neurons in a single cubic millimeter of brain, what amounts to a single voxel in a typical clinical MRI, would represent an amazing achievement in neuroscience. "The finest resolution for [magnetic resonance] now is bigger than the largest thing we can imagine reconstructing."

Josh Sanes, director of the Center for Brain Science, says bridging these different imaging approaches is a major interest for researchers at the center. Optical and neuroimaging approaches in neuroscience are both going to advance in the coming years as more centers and individual researchers like those in Boston push the imaging envelope. But whether MRI one day will reach the resolution of current optical approaches is something only time will tell.

Nathan Blow is the technology editor for Nature and Nature Methods

(n.blow@boston.nature.com). 
SUPPLIERS GUIDE: COMPANIES OFFERING IMAGING SUPPLIES

\section{Company}

4pi Analysis Inc.

Alpha 0mega

Applied Precision

Applied Scientific Instrumentation

Bioptonics

Bioscan

Bruker Daltonics

Chemicon (Millipore)

Chroma Technology

Energy Beam Sciences

EDAX

Electron Microscopy Sciences

Elekta

Evident Technologies

DVC

Gatan, Inc.

GE Healthcare

Hitachi High Technologies America

Hitachi Medical Systems

Intracellular Imaging

Invitrogen

ISS Group

JPK Instruments

JEOL LTD.

Kodak

Leica Microsystems

LightLab

Lightools

Lumafluor Inc.

Magnex Scientific

MRIequip

Nikon

Olympus America

Omega Optical

Optic Solutions Inc.

Optical Imaging

Optronics

0xford Instruments

Photometrics

PicoQuant

Princeton Instruments

Promega

Qioptiq

Rockland

Semrock

SEMTech Solutions, Inc.

ScanMed

Scientific Instruments and Applications

Siemens Healthcare

STS Elionix

T2 Biosystems

Tescan USA

Thermo Fisher Scientific

Toshiba Medical Systems Corporation

Varian Inc.

VayTek

Veeco

\section{Product}

http://www.4pi.com/

http://www.alphaomega-eng.com/

http://www.api.com/

http://www.asiimaging.com/

http://www.bioptonics.com/

http://www.bioscan.com/

http://www.bdal.de/

http://www.chemicon.com/

http://www.chroma.com/

http://www.ebsciences.com/

http://www.edax.com/

http://www.emsdiasum.com/

http://www.elekta.com/

http://www.evidenttech.com/

http://www.dvcco.com/

http://www.gatan.com/

http://www.gehealthcare.com/

http://www.hitachi-hta.com/

http://www.hitachimed.com/

http://www.intracellular.com/

http://www.invitrogen.com/

http://www.iss-group.co.uk/

http://www.jpk.com/

http://www.jeol.com/

http://www.kodak.com/

http://www.leica-microsystems.com/

http://www.lightlabimaging.com/

http://www.lightools.com/

http://www.lumafluor.com/

http://www.magnex.com/

http://www.mriequip.com/

http://www.nikon.com/

http://www.olympusamerica.com/

http://www.omegafilters.com/

http://www.opticsolutions.com/

http://www.opt-imaging.com/

http://www.optronics.com/

http://www.oxford-instruments.com/

http://www.photomet.com/

http://www.picoquant.com/

http://www.piacton.com/

http://www.promega.com/

http://www.qioptiq.com/

http://www.rockland-inc.com/

http://www.semrock.com/

http://www.semtechsolutions.com/

http://www.scanmed.com/

http://www.sia-cam.com/

http://www.medical.siemens.com/

http://www.sts-elionix.com/

http://www.t2biosystems.com/

http://www.tescan-usa.com/

http://www.thermofisher.com/

http://www.toshiba-medical.co.jp/tmd/english/index.html http://www.varian.com/

http://www.vaytek.com/

http://www.veeco.com/ 